\title{
REVIEW
}

\section{Waking up dormant tumors}

\author{
Joyce C Tse $\mathrm{T}^{* 1,2}$ and Raghu Kalluri 1,2,3
}

\begin{abstract}
As appreciation grows for the contribution of the tumor microenvironment to the progression of cancer, new evidence accumulates to support that the participation of stromal cells can extend beyond the local environment. Recently, Elkabets and colleagues demonstrated a systemic interaction between cancer cells and distant bone marrow cells to support the growth of otherwise indolent tumor cells at a secondary site, raising thought-provoking questions regarding the involvement of stromal cells in maintaining metastatic dormancy.
\end{abstract}

\section{Background}

Research in recent years has highlighted the interactions between cancer cells and stromal cells as crucial determinants of cancer progression [1]. Stromal cells of the tumor microenvironment, which includes fibroblasts, endothelial cells, immune cells and more, actively participate in the tumorigenic process and have become important therapeutic targets in cancer [2]. In addition to local stromal interactions within the tumor microenvironment, tumors appear to have a systemic effect that can influence cancer progression, primarily via the stimulation of bone marrow cells [3].

In a previous study 3 years ago, McAllister and colleagues [4] reported that certain tumors (termed 'instigators') can foster the growth of otherwise indolent tumors (termed 'responders') at a distant anatomical site via the activation and recruitment of bone marrow cells. However, the identity of the activated bone marrow cells and their specific contribution had not been determined.

\section{Article}

Researchers from McAllister's group at Brigham and Women's Hospital have now determined the identity of

\footnotetext{
${ }^{*}$ Correspondence: jtse@bidmc.harvard.edu

${ }^{2}$ Harvard Medical School, Division of Matrix Biology, Department of Medicine, Beth Israel Deaconess Medical Center, 330 Brookline Avenue, Dana 771, Boston, MA 02215, USA

Full list of author information is available at the end of the article
}

the activated bone marrow cells responsible for stimulating the growth of otherwise indolent tumor cells and further characterized the impact of the activated bone marrow cells on these responding tumors [5]. In addition, they have identified a molecular mechanism by which the activated bone marrow cells facilitate growth of the responding tumors.

Histological analysis of the responding tumors stimulated by the bone marrow cells demonstrated a greater desmoplastic reaction with increased distribution of alpha-smooth muscle actin-positive myofibroblasts and excess collagen deposition. Elkabets and colleagues [5] determined that the myofibroblasts are not bone marrow derived; thus, the bone marrow cells are not differentiating into myofibroblasts but rather activating the myofibroblasts within the tumor microenvironment.

Fluorescence-activated cell sorting (FACS) analysis of the activated bone marrow cells identified them as a Scal ${ }^{+}$cKit population of hematopoietic progenitors. While this population of bone marrow cells also exists in mice bearing non-instigating tumors, only the Scal ${ }^{+} \mathrm{CKit}$ population from mice bearing instigator tumors can stimulate the growth of responding tumors. Gene expression profiling of the $\mathrm{Sca}^{+}{ }^{+} \mathrm{CKit}$ populations from instigator-bearing mice and non-instigator-bearing mice identified that the bone marrow cells from instigatorbearing mice exhibit increased expression of granulin, a secreted growth factor of the epithelin family.

In the absence of activated bone marrow cells, recombinant granulin alone was able to stimulate the growth of indolent tumor cells with an accompanying desmoplastic reaction. Notably, assessment of granulin expression in human breast cancer samples demonstrated that high granulin expression is significantly correlated with the most aggressive breast tumor subtypes and reduced patient survival.

\section{Viewpoint}

The role of granulin in promoting tumorigenesis has been previously investigated [6] and its potential as a therapeutic target has been explored via the use of a monoclonal antibody against granulin, which was able to inhibit tumor growth in a dose-dependent manner [7]. However, many of these studies have focused on the function of granulin as expressed by cancer cells; the 
study by McAllister's group [5] is the first to identify a stromal contribution of granulin to tumorigenesis.

Activated bone marrow cells expressing granulin are recruited to the tumor microenvironment to stimulate the growth of otherwise indolent tumor cells. Noting the hematopoietic source of granulin, this raises an interesting question as to whether the assessment of granulin levels in the serum may serve as a prognostic marker for relapse in cancer patients. Additionally, one might also draw a parallel between the indolent responding tumors of these experiments and the dormant metastases of cancer patients. In this case, is bone marrow-derived granulin one of the factors responsible for waking up dormant metastases?

Osteopontin was previously identified as the molecule secreted by the instigator tumor, which activates the bone marrow cells [4]. Is the continued presence of the instigator tumor necessary to maintain activation of the bone marrow cells? Or do the bone marrow cells remain activated despite removal of the instigator tumor? The answers to these questions will have implications on the therapeutic efficacy of primary tumor resection and additional measures that may need to be taken in order to suppress metastatic outgrowth. Even after primary tumor resection, there may be other sources of osteopontin or additional mechanisms that activate bone marrow cells. A greater understanding of the mechanisms underlying bone marrow activation may have important clinical implications for maintaining metastatic dormancy.

Acknowledgements

Work in the authors' laboratory is supported by the Champalimaud Metastasis Research Programme, NIH grants CA125550, CA155370, CA151925, and
DK081576. JCT is supported by the Department of Defense Breast Cancer Research Program Predoctoral Traineeship Award.

\section{Competing interests}

The authors declare that they have no competing interests.

\section{Author details}

${ }^{1}$ Harvard Medical School, Boston, MA 02115, USA. 2Division of Matrix Biology, Department of Medicine, Beth Israel Deaconess Medical Center, 330 Brookline Avenue, Dana 771, Boston, MA 02215, USA. ${ }^{3}$ Harvard-MIT Division of Health Sciences and Technology, Boston, MA 02115, USA.

Published: 10 June 2011

\section{References}

1. Wels J, Kaplan RN, Rafii S, Lyden D: Migratory neighbors and distant invaders: tumor-associated niche cells. Genes Dev 2008, 22:559-574.

2. Joyce JA: Therapeutic targeting of the tumor microenvironment. Cancer Cell 2005, 7:513-520.

3. MCAllister SS, Weinberg RA: Tumor-host interactions: a far-reaching relationship. J Clin Oncol 2010, 28:4022-4028.

4. McAllister SS, Gifford AM, Greiner AL, Kelleher SP, Saelzler MP, Ince TA, Reinhardt F, Harris LN, Hylander BL, Repasky EA, Weinberg RA: Systemic endocrine instigation of indolent tumor growth requires osteopontin. Cell 2008, 133:994-1005.

5. Elkabets M, Gifford AM, Scheel C, Nilsson B, Reinhardt F, Bray MA, Carpenter AE, Jirström K, Magnusson K, Ebert BL, Pontén F, Weinberg RA, McAllister SS: Human tumors instigate granulin-expressing hematopoietic cells that promote malignancy by activating stromal fibroblasts in mice. J Clin Invest, 121:784-799.

6. Ong CH, Bateman A: Progranulin (granulin-epithelin precursor, PC-cell derived growth factor, acrogranin) in proliferation and tumorigenesis. Histol Histopathol 2003, 18:1275-1288.

7. Ho JC, Ip YC, Cheung ST, Lee YT, Chan KF, Wong SY, Fan ST: Granulin-epithelin precursor as a therapeutic target for hepatocellular carcinoma. Hepatology 2008, 47:1524-1532.

doi: $10.1186 /$ bcr2872

Cite this article as: Tse JC, Kalluri R: Waking up dormant tumors. Breast

Cancer Research 2011, 13:310. 